\title{
Oribatid mites (Acari: Oribatida) of the Isle of Man revisited
}

\author{
F.D. Monson ${ }^{1} \&$ M. Luxton ${ }^{2}$ \\ ${ }^{1}$ National Museums Liverpool (Entomology Department), William Brown St., Liverpool L3 8EN \\ ${ }^{2} 118$ Merthyr Street, Barry, Vale of Glamorgan CF63 4LD \\ E-mail: frank.monson@gmail.com
}

\begin{abstract}
This paper is based on a collection of oribatid mites from the Isle of Man, British Isles that was made during a survey of the island conducted in 1988. A total of 172 species and one subspecies of oribatid mites has now been recorded for the Isle of Man, in comparison with 363 species for the whole of Britain. Twelve of the species and one subspecies reported herein are new to Britain, namely: Ceratozetes conjunctus, Ceratozetes sp., Liochthonius alpestris, Neoliochthonius globuliferus, Oppiella (Oppiella) splendens, Oribatula longilamellata, Pantelozetes berlesei, Planoristes acuspidatus, Scheloribates minifimbriatus, Serratoppia serrata, Suctobelbella sp., Tectocepheus velatus alatus and Zygoribatula propinqua. A checklist of all oribatid mites found to date in the Isle of Man is provided.
\end{abstract}

\section{INTRODUCTION}

The acarine suborder Oribatida (also called Cryptostigmata) is one of the most numerically dominant arthropod groups in the organic horizons of most soils. Globally, over 10,000 species have been described (Subías, 2019), which is considered to represent one third to one tenth of the true diversity (Walter \& Proctor, 2013; Behan-Pelletier \& Lindo, 2019). Monson (2011) provides a brief introduction to British oribatid mites.

Previously published records for the Isle of Man can be found in Luxton (1987). The present study was based on material collected by M.L. in 1988. The collection forms part of the Luxton Archive given to the National Museums Liverpool on M.L.'s retirement.

This paper is intended to update and expand Luxton's 1987 account, and provides a checklist of all oribatid mite species found to date in the Isle of Man.

\section{MATERIALS \& METHODS}

A total of 135 samples was collected from 36 locations. The samples were confined to small polythene bags for transport to the extractor in Liverpool. The necks of the bags were tied to conserve moisture and small paper tags were looped around the consequent knots by their attaching string. The paper tags were labelled fully, in pencil, in the field. Thus, on transferring the sample to the extractor, they could be pulled free of the string and used in the collecting vessel obviating the need to write a second label. Banks of Tullgren funnels were used for extraction over a period of one day for each extraction and the catch was collected in a $70 \%$ glycerol mixture in plastic pots. On completion of extraction, the volume of liquid in the pot was tripled by the addition of absolute alcohol and a snap on lid secured tightly to prevent evaporation. Specimens were examined in a stereomicroscope and compound light microscope when temporarily mounted in $100 \%$ lactic acid on glass cavity slides at room temperature. For species new to Britain, specimen counts were made. Identified specimens were stored in $70 \%$ alcohol and deposited in National Museums Liverpool (Entomology Department).

\section{SITES STUDIED (see Fig.1)}

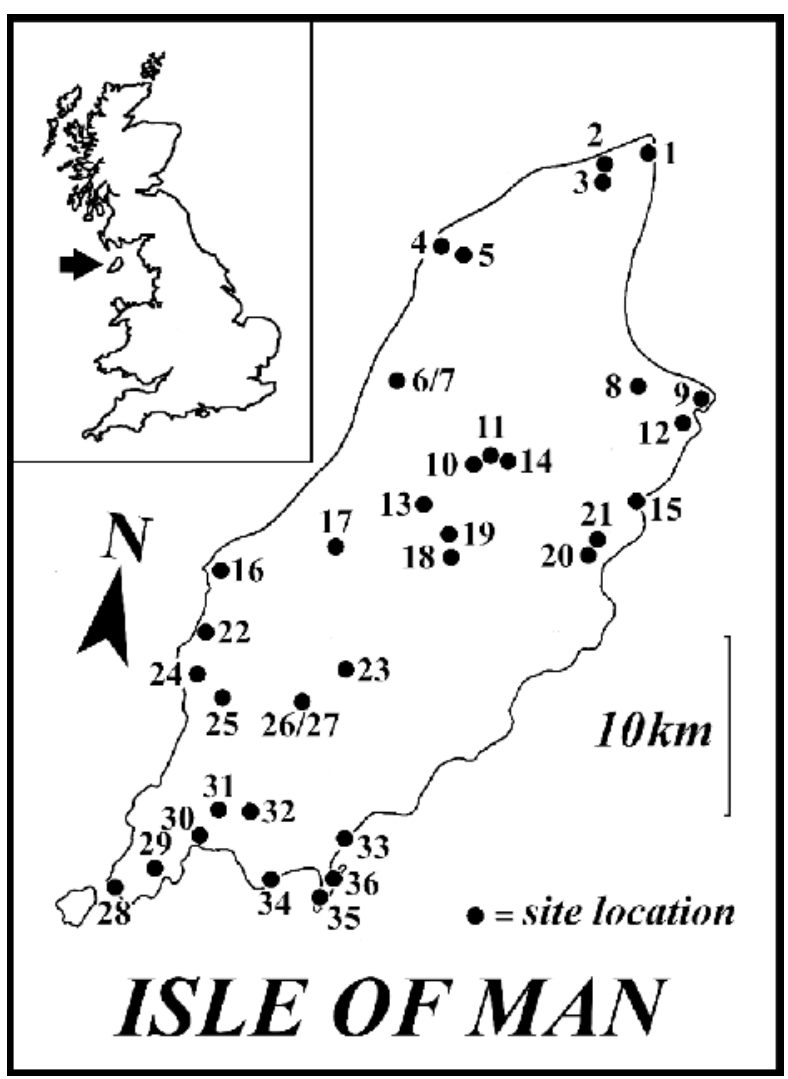

Fig. 1. Location of the Isle of Man (arrowed in the inset map of Britain) and sites where samples were collected.

Site 1. Point of Ayre, 21.4.88 (NX468047): Sample 652, gorse (Ulex) litter. Sample 654, marram grass (Ammophila) litter. Sample 658, lichen (Xanthoria parietina) from concrete of old gun emplacement. 
Sample 662, moss and heather (Calluna) litter. Sample 665 , moss on sand.

Site 2. The Ayres, near Visitor Centre, 17.4.88 (NX439039): Sample 618, dry heather (Calluna) litter. Sample 625, gorse (Ulex) litter. Sample 695, lichen (unidentified) on sand. Sample 709, moss on sand.

Site 3. Manx National Trust (New Site), The Ayres, 21.4.88: Sample 656 (NX433035), bracken (Pteridium) litter. Sample 660 (NX432032), grass turf in wet slack. Sample 663 (NX435035), moss beneath pine (Pinus). Sample 671 (NX432032), moss (Sphagnum) from a wet slack. Sample 676 (NX432032), mossy turf in wet slack. Sample 682 (NX435035), pine (Pinus) litter.

Site 4. Sartfield, 21.4.88 (SC353997): Sample 667, moss on dunes.

Site 5. Ballaugh Curraghs near Ballaugh, 21.4.88 (SC365956): Sample 647, thin, dryish moss from willow (Salix) trunk. Sample 655, moss (Sphagnum) on ground. Sample 657, from lichen (Parmelia saxatilis) on willow trunk. Sample 666, dryish willow (Salix) litter. Sample 669, grass (Molinia) litter. Sample 670, waterlogged willow (Salix) litter.

Site 6. Orrisdale, near Kirk Michael, 22.4.88 (SC329928): Sample 653, moss on asbestos roof. Sample 678, beech (Fagus) litter. Sample 688, moss on ground beneath trees.

Site 7. Bishopscourt Glen, near Kirk Michael, 22.4.88: Sample 691 (SC331924), mixed deciduous litter. Sample 736 (SC331924), woody plant (Rhododendron) and palm litter. Sample 748 (SC333922), moss (Sphagnum) on soil bank.

Site 8. Ballure Plantation, 17.4.88 (SC454923): Sample 624, moss (Polytrichum) from above drainage ditch. Sample 633, spruce (Picea) litter. Sample 703, lichen (unidentified) and soil from above drainage ditch opposite Ballure Plantation. Sample 716, moss on tree stump.

Site 9. Maughold, 17.4.88 (SC493917): Sample 620, moss on stone wall.

Site 10. Ballaugh Plantation, near Ballaugh, 21.4.88 (SC367912): Sample 651, pine (Pinus) litter.

Site 11. Tholt-e-Will Glen, 21.4.88: Sample 645 (SC378891), blue pine (Pinus wallichiana) litter. Sample 646 (SC378890), moss on slate wall. Sample 648 (SC378891), moss (Polytrichum) on ground beneath pine (P. wallichiana). Sample 649 (SC377888), heather (Calluna) litter on moor opposite Tholt-e-Will Glen. Sample 661 (SC378891), moss on tree stump. Sample 664 (SC378891), very wet liverworts/moss on soil bank. Sample 677 (SC377888), moss among heather, opposite Tholt-e-Will Glen.
Site 12. Dreemskerry Quarry, 17.4 .88 (SC477911): Sample 710, wet moss on cliff.

Site 13. Sartfell Plantation, 19.4.88: Sample 619 (SC344866), moss beneath heather (Calluna) on bank at edge of pine (Pinus) stand. Sample 636 (SC344866), moss (Sphagnum) from drainage ditch at edge of Plantation. Sample 705 (SC344866), tree (Thuja/Cupressus) litter. Sample 707 (SC344865), from wet heather (Calluna) litter and moss from ground local to Plantation. Sample 746 (SC344866), pine (Pinus) litter.

Site 14. Snaefell near The Bungalow, 21.4.88 (SC387880): Sample 650, grass turf. Sample 668, very wet moss on rock face.

Site 15. Glen Dhoon, 19.4.88 (SC455865): Sample 629, liverworts on soil bank. Sample 630, thin, slimy moss/liverworts on tree stump. Sample 631, mixed deciduous leaf litter. Sample 699, moss on ash trunk. Sample 751, moss on soil bank.

Site 16. The Raggett, near Peel, 18.4.88 (SC244830): Sample 617, larch (Larix) litter. Sample 621 gorse (Ulex) litter. Sample 622, mixed tree litter. Sample 639, moss on sycamore trunk. Sample 693, moss on soil beneath sycamore. Sample 698, oak (Quercus) litter. Sample 702, moss on rocks. Sample 732, grass and bracken (Pteridium) litter.

Site 17. Glen Helen, 23.4.88 (SC298843): Sample 640, larch (Larix) litter. Sample 700, moss on disintegrating tree stump. Sample 725, beech (Fagus) litter. Sample 735 , moss on fallen log. Sample 742, moss on slate. Sample 744, liverworts on bank of soil on slate. Sample 749 , moss on beech (Fagus) trunk. Sample 750, thick moss (Sphagnum) on soil bank.

Site 18. Colden Plantation, 19.4.88 (SC358837): Sample 641 , moss on stone, edge of Norway spruce (Picea abies) stand. Sample 712, moss on tree stump. Sample 713, tree (Abies) litter.

Site 19. Injebreck, 19.4.88 (SC356848): Sample 634, moss (Homalothecium sericeum) on face of wall. Sample 635, wet moss on trunk of beech (Fagus). Sample 734, moss (Polytrichum) on top of wall. Sample 745, mixed deciduous beech/sweet chestnut (Fagus/Castanea) litter.

Site 20. Axnfell Plantation, near Laxey, 19.4.88 (SC423838): Sample 637, lichen on larch (Larix) trunk. Sample 706, moss on ground beneath larch (Larix). Sample 721, moss mound on old stone building. Sample 722, gorse (Ulex) litter. Sample 737, pine (Pinus) litter.

Site 21. Laxey Glen Gardens, Laxey, 19.4 .88 (SC430843): Sample 627, beech (Fagus) litter. Sample 628 , moss on bridge parapet. Sample 638, moss on ground beneath woody plant (Rhododendron). Sample 723 , finely divided liverworts in running water on bank. 
Sample 726, liverworts at edge of drainage ditch. Sample 729, moss on beech (Fagus) trunk.

Site 22. Glen Maye, 18.4.88 (SC233798): Sample 626, sycamore litter. Sample 704, moss on soil at edge of gorge. Sample 711, deep wet moss on wall of gorge near stream. Sample 715, liverworts on soil at edge of gorge. Sample 718, liverworts on rock.

Site 23. Archallagan Plantation, 23.4 .88 (SC300780): Sample 724, moss on floor of pine (Pinus) stand. Sample 741, pine litter.

Site 24. Niarbyl, Elby Point, 20.4.88 (SC211777): Sample 643, lichen (X. parietina). Sample 686, thrift clump (Armeria maritima) on cliff. Sample 717, moss clumps on cliff face.

Site 25. Slieu Mooar Plantation, 18.4.88 (SC234764): Sample 616, moss on fallen spruce (Picea) trunk. Sample 632, spruce (Picea) litter. Sample 694, moss (Galium saxatile) and plant at edge of spruce (Picea) forest.

Site 26. South Barrule Plantation, 18.4.88 (SC277761): Sample 623, spruce (Picea) litter. Sample 692, moss (Sphagnum) in drainage ditch.

Site 27. Barrule Plantation, 18.4.88 (SC277761): Sample 719, wet moss beneath (Calluna) and young tree (Abies).

Site 28. Spanish Head, 20.4.88 (SC173667): Sample 642, lichen (Ramalina siliquosa), on cliff top. Sample 674, gorse (Ulex) litter on cliff top. Sample 730, moss and powdery lichen on soil of cliff top. Sample 733, moss (Polytrichum?) on cliff top. Sample 743, grass clump in rock cleft.

Site 29. The Howe, 20.4.88 (SC198678): Sample 747, moss on wall.

Site 30. Ballagawne, 18.4.88 (SC223695): Sample 714, moss on wall opposite churchyard.

Site 31. Colby, 18.4.88 (SC232706): Sample 697, moss on wall.

Site 32. Ballabeg, 20.4.88 (SC247704): Sample 690, moss on wall.

Site 33. Cass-ny-Hawin Head, 20.4 .88 (SC297693): Sample 683, moss on rock on slopes of inlet. Sample 689, gorse (Ulex) litter on cliff top at inlet. Sample 701, oak (Quercus) litter on slope of inlet. Sample 720, moss on oak (Quercus) trunk on slopes of inlet. Sample 740, bracken (Pteridium) litter on slope of inlet.

Site 34. Castletown (near), 18.4.88 (SC263682): Sample 696 , moss on wall.

Site 35. Langness, 20.4.88 Sample 659 (SC282654), lichen (X. parietina) on conglomerate rock. Sample 672
(SC285658), heather (Calluna) litter on slate outcrop. Sample 673 (SC285658), lichen ( $R$. siliquosa) on conglomerate rock. Sample 679 (SC283662), saltmarsh turf. Sample 680 (SC285658), moss on slate outcrop. Sample 681 (SC285 658), green/grey thallate lichen ( $P$. saxatilis?) on slate outcrop. Sample 684 (SC285658), marine lichen (Lichina pygmaea) on conglomerate rock. Sample 687 (SC285658), gorse (Ulex) litter on slate outcrop. Sample 708 (SC282654), plant (Armeria) clump on conglomerate rock. Sample 727 (SC285658), gorse (Ulex) litter on slate outcrop. Sample 728 (SC282654), moss in damp area of conglomerate rock at top of shore. Sample 731 (SC285658), moss on slate outcrop. Sample 739 (SC285658), lichen (R. siliquosa) on slate.

Site 36. St. Michael's Isle, 20.4.88 (SC296673): Sample 644 , lichen (X. parietina), on coastal rocks. Sample 675, lichen $(R$. siliquosa) on rock. Sample 685, plant (Sedum/Armeria) clump on rock. Sample 738 (SC296674) tidal debris, top of shore.

\section{RESULTS}

Unless otherwise stated, superfamily and family organisation are in accordance with Schatz et al. (2011) and lower level taxonomy is mainly in accordance with Weigmann (2006). In the text below "Britain" is used in the watsonian sense to refer to the island of Great Britain and its offshore islands, including the Isle of Man (Watson, 1852).

* = species listed in Luxton (1987)

$\dagger=$ species collected in 1988, new to Britain (bold text)

$\wedge=$ species collected in 1988, not new to Britain

Each specific name is followed by sampling site number(s) and, as appropriate, historical notes.

\section{Overview of oribatid mites found in the Isle of Man}

Total number of species recorded $=172$

Number of subspecies $=1$

Number of species with genus assignation only $=2$

Number of genera $=85$

Number of families $=41$

Number of species listed in Luxton $(1987)=60($ not 59 as stated in Luxton, 1987)

Number of species new to the Isle of Man found in the 1988 collection $=112$

Number of species new to Britain found in the 1988 collection $=12$

\section{Checklist of oribatid mites found in the Isle of Man}

Suborder Oribatida van der Hammen, 1968

Infraorder Enathronota Grandjean, 1969

Superfamily Brachychthonioidea Thor, 1934

Family Brachychthoniidae Thor, 1934

Genus Brachychthonius Berlese, 1910

*Brachychthonius berlesei Willmann, 1928

Genus Liochthonius van der Hammen, 1952

$\uparrow$ Liochthonius alpestris (Forsslund, 1958) (622, $638,663,696,709)$ (62 specimens). 
Distribution: Palaearctic (Subías, 2019); Europe (Weigmann, 2006).

*^L. brevis (Michael, 1888) (623, 633, 640, 656, 663, 665, 671, 682, 687, 689, 694, 705, 706, 707, 722, 741, 746, 751).

^L. clavatus (Forsslund, 1942) (747).

$\wedge$ L. lapponicus (Trägårdh, 1910) (671).

${ }^{\wedge}$ L. perfusorius Moritz, 1976 (633, 694, 706, 707, 722, 741, 751).

${ }^{\wedge}$ L. sellnicki (Thor, 1930) (622).

${ }^{\wedge}$ L. strenzkei Forsslund, 1963 (622).

Genus Neoliochthonius Lee, 1982

$\uparrow$ Neoliochthonius globuliferus (Strenzke, 1951) (707) (one specimen).

Distribution: Central Europe and Mexico (Subías, 2019); Europe (Weigmann, 2006).

${ }^{\wedge} N$. piluliferus (Forsslund, 1942) (696).

Genus Poecilochthonius Balogh, 1943

^ Poecilochthonius spiciger (Berlese, 1910) (669).

Genus Sellnickochthonius Krivolutsky, 1964

^Sellnickochthonius cricoides (Weis-Fogh, 1948)

(654, 656, 671).

${ }^{\wedge} S$. honestus (Moritz, 1976) (648).

${ }^{\wedge} S$. rostratus (Jacot, 1936) (622).

Note: $S$. rostratus is sometimes synonymised with $S$. hungaricus subsp. rostratus, although Weigmann (2006) regards it as a separate species.

Genus Synchthonius van der Hammen, 1952

*^Synchthonius crenulatus (Jacot, 1938) (671).

Superfamily Hypochthonioidea Berlese, 1910

Family Eniochthoniidae Grandjean, 1947

Genus Eniochthonius Grandjean, 1933

${ }^{\wedge}$ Eniochthonius minutissimus (Berlese, 1903) (640, $740,748)$.

Family Hypochthoniidae Berlese, 1910 Genus Hypochthonius C.L. Koch, 1835

*^Hypochthonius rufulus C.L. Koch, 1835 (617, 622, 623, 638, 640, 648, 664, 694, 703, 706, 725, $732,736,740,741)$

Infraorder Mixonomata Grandjean, 1969

Superfamily Eulohmannioidea Grandjean, 1931

Family Eulohmanniidae Grandjean, 1931

Genus Eulohmannia Berlese, 1910

^ Eulohmannia ribagai (Berlese, 1910) (638).

Superfamily Euphthiracaroidea Jacot, 1930

Family Euphthiracaridae Jacot, 1930

Genus Acrotritia Jacot, 1923

^Acrotritia duplicata (Grandjean, 1953) (640,713).

Superfamily Phthiracaroidea Perty, 1841

Family Phthiracaridae Perty, 1841

Genus Phthiracarus Perty, 1841

*Phthiracarus affinis (Hull, 1914)

${ }^{\wedge} P$. ferrugineus (C.L. Koch, 1841) $(622,655,666$, 670).

*P. longulus (C.L. Koch, 1841)

${ }^{\wedge} P$. montanus C. Pérez-Íñigo, 1969 (622, 623, 626, 627, 631, 640, 651, 661, 672, 711, 729, 735, 737, 749).
Genus Steganacarus Ewing, 1917

^Steganacarus (Steganacarus) Ewing, 1917

${ }^{\wedge}$ Steganacarus. (Steganacarus) magnus

(Nicolet, 1855) (626, 627, 628, 631, 640, 648, 662, 674, 678, 682, 687, 689, 691, 701, 703, 706, 713, $725,727,736,737,740,748)$.

Infraorder Desmonomata Woolley, 1973

Hyporder Nothrina van der Hammen, 1982

Superfamily Crotonioidea Thorell, 1876

Family Crotoniidae Thorell, 1876

Genus Camisia von Heyden, 1826

${ }^{\wedge}$ Camisia biurus (C.L. Koch, 1839) (618, 619, 662, 707).

${ }^{\wedge}$ C. horrida (Hermann, 1804) $(620,652,690,696$, 733).

${ }^{\wedge}$ C. invenusta (Michael, 1888) $(646,667,681,743)$.

${ }^{\wedge}$ C. segnis (Hermann, 1804) $(618,619,646,652$, $662,698,712,731)$.

${ }^{\wedge}$ C. spinifer (C.L. Koch, 1835) $(672,694,706,708$, 722, 727, 731, 737, 741, 743).

Genus Platynothrus Berlese, 1913

*^ Platynothrus peltifer (C.L. Koch, 1839) (619, $621,622,623,624,640,648,649,650,655,666$, $668,669,670,677,692,700,707,710,712,713$, 723, 725, 726, 737, 741, 748).

${ }^{\wedge} P$. thori (Berlese, 1904) (670).

Family Hermanniidae Sellnick, 1928

Genus Hermannia Nicolet, 1855

*^Hermannia gibba (C.L. Koch, 1839) $(617,622$, 626, 627, 631, 640, 659, 688, 689, 700, 701, 711, 715, 717, 718, 732, 735, 736, 740, 748, 749).

${ }^{\wedge} H$. pulchella Willmann, 1952 (679).

*^ $H$. reticulata Thörell, $1871(618,620,624,625$, 646, 652, 654, 661, 668, 672, 685, 686, 703, 705, 709, 716, 731, 741, 745).

Family Malaconothridae Berlese, 1916

Genus Malaconothrus Berlese, 1904

*Malaconothrus monodactylus (Michael, 1888)

Genus Tyrphonothrus Knülle, 1957

*Tyrphonothrus maior (Berlese, 1910) (as

Trimalaconothrus novus in Luxton, 1987).

Family Nanhermanniidae Sellnick, 1928

Genus Nanhermannia Berlese, 1913

*^Nanhermannia coronata Berlese, 1913 (624, 631, 649, 651, 661, 707, 719, 737, 741, 746).

${ }^{\wedge} N$. elegantula Berlese, 1913 (629, 713, 722, 723, 740).

*N. sellnicki Forsslund, 1958

Family Nothridae Berlese, 1896

Genus Nothrus C.L. Koch, 1835

${ }^{\wedge}$ Nothrus anauniensis Canestrini \& Fanzago, 1876 (719).

${ }^{* \wedge} N$. palustris C.L. Koch, $1839(617,622,659,691$, $715,748)$.

${ }^{\wedge} N$. silvestris Nicolet, $1855(623,624,633,640,648$, 649, 651, 656, 662, 671, 685, 703, 707, 722, 740, 748). 
Family Trhypochthoniidae Willmann, 1931

Genus Trhypochthonius Berlese, 1904

${ }^{\wedge}$ Trhypochthonius tectorum (Berlese, 1896) (733, 739).

Hyporder Brachypylina Hull, 1918

Superfamily Neoliodoidea Sellnick, 1928

Family Neoliodidae Sellnick, 1928

Genus Poroliodes Grandjean 1934

^ Poroliodes farinosus (C.L. Koch, 1840) (683).

Superfamily Damaeoidea Berlese, 1896

Family Damaeidae Berlese, 1896

Genus Belba von Heyden, 1826

${ }^{\wedge}$ Belba corynopus (Hermann, 1804) (627).

Genus Damaeus C.L. Koch, 1835

${ }^{\wedge}$ Damaeus (Adamaeus) onustus (C.L. Koch, 1844)

(627, 701, 732).

${ }^{\wedge} D$. (Damaeus) riparius Nicolet, 1855 (617).

${ }^{\wedge}$ D. (Paradamaeus) clavipes (Hermann, 1804) (636, 647, 674, 716).

Genus Kunstidamaeus Miko, 2006

${ }^{\wedge}$ Kunstidamaeus tenuipes (Michael, 1885) (734).

Genus Metabelba Grandjean, 1936

${ }^{\wedge}$ Metabelba papillipes (Nicolet. 1855) (617, 622, 689).

Genus Porobelba Grandjean, 1936

^Porobelba spinosa (Sellnick, 1920) $(618,624,634$, 640, 654, 656, 662, 671, 703, 706, 711, 714, 732, $734,748)$.

Superfamily Cepheoidea Berlese, 1896

Family Cepheidae Berlese, 1896

Note: The name Cepheidae is a junior homonym of Cepheidae Agassiz, 1862 (Schatz et al., 2011). Halliday \& Norton (2019) have made a proposal to the International Commission of Zoological Nomenclature to emend the name; we retain the traditional spelling pending approval.

Cepheus C.L. Koch, 1835

${ }^{\wedge}$ C. latus C.L. Koch, 1835 (623, 637).

Superfamily Zetorchestoidea Michael, 1898

Family Eremaeidae Oudemans, 1900

Genus Eueremaeus Mihelčič, 1963

*^Eueremaeus oblongus (C.L. Koch, 1835) (618, $620,630,634,652,654,662,675,683,685,696$, $700,711,714,717,720,730,743,749$ ) (as Eremaeus oblongus in Luxton, 1987).

Genus Tricheremaeus Berlese, 1908

*Tricheremaeus serratus (Michael, 1885) (as Tricheremaeus serrata in Luxton, 1987).

Superfamily Gustavioidea Oudemans, 1900

Family Astegistidae Balogh, 1961

Genus Cultroribula Berlese, 1908

${ }^{\wedge}$ Cultroribula bicultrata Berlese, 1905 (740).

${ }^{* \wedge}$ C. juncta (Michael, 1885) $(624,711)$.

Family Gustaviidae Oudemans, 1900

Genus Gustavia Kramer, 1979

*^Gustavia microcephala (Nicolet, 1855) (622, 686, 732).
Family Liacaridae Sellnick, 1928

Genus Adoristes Hull, 1916

^Adoristes ovatus (C.L. Koch, 1839) (617, 622, 623, 624, 625, 640, 641, 645, 647, 652, 667, 669, 683, 687, 689, 697, 701, 710, 711, 712, 713, 722, 724, 727, 731, 732, 737, 740, 741)

*A. poppei (Oudemans, 1906)

Genus Liacarus Michael, 1898

${ }^{\wedge}$ Liacarus coracinus (C.L. Koch, 1841) (669).

${ }^{\wedge}$ L. subterraneus (C.L. Koch, 1841) (732).

Genus Planoristes Iturrondobeitia \& Subías, 1978

$\uparrow$ Planoristes acuspidatus Iturrondobeitia \& Subías, 1978 (633) (one specimen).

Distribution: Spain (Subías, 2019).

Genus Xenillus Robineau-Desvoidy, 1839

*^Xenillus tegeocranus (Hermann, 1804) (617, 621, 622, 626, 640, 645, 647, 672, 674, 682, 685, 687, 689, 691, 701, 711, 727, 732, 736, 740, 748, 749).

Family Peloppiidae Balogh, 1943

Genus Ceratoppia Berlese, 1908

*^Ceratoppia bipilis (Hermann, 1804) (619, 622, 623, 626, 632, 633, 636, 640, 645, 648, 652, 654, $656,663,666,669,671,674,678,687,689,701$, 706, 710, 713, 714, 715, 716, 719, 722, 724, 727, $740,741,748,749)$.

Superfamily Carabodoidea C.L. Koch, 1837

Family Carabodidae C.L. Koch, 1837

Genus Carabodes C.L. Koch, 1835

${ }^{\wedge}$ Carabodes labyrinthicus (Michael, 1879) (675, 681, 708, 731, 739, 749, 751).

*^C. marginatus (Michael, 1884) (624, 645, 661, 662, 671, 672, 680, 685, 699, 703, 720, 731, 734, $745,749)$.

*^C. willmanni Bernini, 1975 (618, 624, 632, 635, $645,660,661,662,665,668,671,672,673,677$, $680,685,695,703,708,730,731,733,739,741)$.

Genus Odontocepheus Berlese, 1913

*^Odontocepheus elongatus (Michael, 1879) (631, $640,648,656,672,706,720,727,740,741,748)$.

Superfamily Oppioidea Grandjean, 1951

Family Autognetidae Grandjean, 1960

Genus Autogneta Hull, 1916

^Autogneta parva Forsslund, 1947 (749).

Family Oppiidae Grandjean, 1951

Genus Berniniella J. Balogh, 1983

^ Berniniella bicarinata (Paoli 1908) (662, 663, 682).

*^B. sigma (Strenzke, 1951) $(656,689,713,746)$.

Genus Dissorhina Hull, 1916

*^Dissorhina ornata (Oudemans, 1900) $(617,618$, $619,620,622,624,633,634,636,637,638,640$, $645,647,648,649,650,651,652,654,655,661$, $662,663,664,665,666,667,669,671,672,674$, 679, 680, 682, 685, 686, 687, 689, 690, 691, 694, 695, 700, 701, 703, 704, 705, 706, 707, 708, 709, $710,711,713,714,715,716,719,721,722,725$, 730, 734, 735, 736, 737, 741, 742, 743, 744, 748, 750).

Genus Microppia Balogh, 1983

^Microppia minus (Paoli, 1908) (650). 
Genus Multioppia Hammer, 1961

*^Multioppia neglecta C. Pérez-Íñigo, 1969 (621, 622, 633, 654, 656, 663, 669, 672, 674, 680, 682, 685, 686, 687, 689, 691, 696, 706, 711, 716, 724, $727,732,737,748)$.

Genus Oppiella Jacot, 1937

Subgenus Oppiella (Moritzoppia) Subías \& Rodríguez, 1988

*^Oppiella (Moritzoppia) keilbachi (Moritz, 1969) $(618,652,654,662,706,711,735,749)$ (as Moritziella keilbachi in Luxton, 1987).

*^O. (M.) neerlandica (Oudemans, 1900) $(617,619$, $622,623,629,631,632,636,640,645,648,652$, 655, 664, 666, 669, 685, 694, 700, 710, 725, 742, $744,748,750$ ) (as Lauroppia neerlandica in Luxton, 1987).

*^O. (M.) unicarinata (Paoli, 1908) $(635,645,664$, $672,729,734,735$ ) (as Moritziella unicarinata in Luxton, 1987).

Subgenus Oppiella (Oppiella) Jacot, 1937

^Oppiella (Oppiella) falcata (Paoli, 1908) (706).

^ O. (O.) maritima (Willmann, 1929) (644, 661, 748, $749)$.

*^O. (O.) nova (Oudemanns, 1902) $(616,623,624$, $638,648,654,662,663,664,665,671,682,703$, 706, 724, 741, 748) (as Oppiella nova in Luxton, 1987).

^O. (O.) propinqua Mahunka \& Mahunka-Papp, $2000(631,725)$.

†Oppiella (Oppiella) splendens (C.L. Koch, 1841) $(630,634,668,699,700,703,720,729,735,749)$ (numerous specimens).

Distribution: Holarctic (Weigmann, 2006) and Australia (Subías, 2019).

Subgenus Oppiella (Rhinoppia) Balogh, 1983

*^Oppiella (R.) obsoleta (Paoli, 1908) (617, 640, 748) (as Medioppia obsoleta in Luxton, 1987).

${ }^{\wedge} O$. (R.) subpectinata (Oudemans, 1900) $(619,622$, 633, 640, 645, 648, 649, 663, 664, 666, 669, 677, 680, 689, 694, 695, 696, 705, 706, 710, 713, 716, 719, 722, 736, 740, 741, 746, 748).

Genus Ramusella Hammer, 1962

${ }^{\wedge}$ Ramusella assimilis (Mihelčič, 1956) (671, 731, 741).

${ }^{\wedge}$ R. clavipectinata (Michael, 1885) (650, 663, 667, 669, 671, 672, 674, 680, 682, 727, 731).

${ }^{\wedge} R$. elliptica (Berlese, 1908) (626, 633, 663, 665, 672, 674, 709, 731).

${ }^{\wedge}$ R. insculpta (Paoli, 1908) $(674,687)$.

Genus Serratoppia Subías \& Mínguez, 1985

†Serratoppia serrata (Mihelčič, 1956) (663, 682, 689) (three specimens).

Distribution: Western Palaearctic (except North) and Venezuela (Subías, 2019).

Family Quadroppiidae Balogh, 1983

Genus Coronoquadroppia Ohkubo, 1995

Note: The genus Coronoquadroppia Okhubo, 1995 was not regarded by Weigmann (2006) as being sufficiently distinct from Quadroppia Jacot, 1939, but was accepted by that author in a subsequent publication (Weigmann, 2010).
*^Coronoquadroppia galaica Mínguez, Ruis \& Subías, 1985 (624, 633, 638, 656, 672, 680, 689, 706, 711, 713, 727, 737, 744, 748) (as Quadroppia bellula in Luxton, 1987).

${ }^{\wedge}$ C. monstruosa (Hammer, 1979) (721).

${ }^{\wedge}$ C. pseudocircumita Mínguez, Ruis \& Subías, 1985 $(638,706,740,746)$.

Genus Quadroppia Jacot, 1939

*^Quadroppia maritalis Lions, 1982 (633, 638, 640, 706, 713, 741, 746).

${ }^{* \wedge} Q$. quadricarinata (Michael, 1885) (618, 619, 628, 642, 646, 689, 690, 696, 702, 708, 709, 713, 721, 734, 735, 744, 746, 747, 749).

Family Thyrisomidae Grandjean, 1953

Genus Banksinoma Oudemans, 1930

*^Banksinoma lanceolata (Michael, 1885) (619, 624, 629, 633, 645, 648, 654, 656, 661, 662, 669, 672, 680, 687, 689, 693, 694, 701, 705, 710, 713, 716, 722, 727, 731, 737, 744).

Genus Pantelozetes Grandjean, 1953

$†$ Pantelozetes berlesei Fujikawa, 1979 (622) (one specimen).

Distribution: Southwestern Europe (Subías, 2019).

^P. paolii (Oudemans, 1913) (622, 651, 669, 678, $682)$.

Superfamily Trizetoidea Ewing, 1917

Family Suctobelbidae Jacot, 1938

Genus Suctobelba Paoli, 1908

${ }^{\wedge}$ Suctobelba regia Moritz, 1970 (634, 638, 699, 701, 710, 729, 735, 748) (eight specimens).

*^S. trigona (Michael, 1888) $(618,628,633,638$, 641, 644, 648, 649, 672, 680, 682, 689, 710, 711, 712, 721, 737, 742, 744, 747, 748, 749).

Genus Suctobelbella Jacot, 1937

^Suctobelbella acutidens (Forsslund, 1941) (617, 618, 633, 638, 648, 654, 656, 663, 672, 682, 687, 689, 691, 706, 709, 712, 723, 732, 737, 742, 744, $748,751)$.

^S. arcana Moritz, 1970 (707, 740, 746, 751).

${ }^{\wedge} S$. falcata (Forsslund, 1941) (706).

^S. hamata Moritz, 1970 (706, 737).

*^S. nasalis (Forsslund, 1941) (633, 689, 727, 732,

740, 749).

^ S. perforata (Strenzke, 1950) $(648,751)$.

*^S. sarekensis Forsslund, 1941 (618, 645, 689, 671, 706, 713, 737, 748).

${ }^{\wedge} \mathrm{S}$. similis (Forsslund, 1941) $(648,652,689,746$, 748, 751).

*^S. subcornigera (Forsslund, 1941) $(633,638,645$, $648,656,663,665,669,672,680,682,685,689$, 703, 706, 707, 709, 711, 713, 722, 727, 737, 740, 742, 744, 748, 749, 751).

${ }^{\wedge} S$. subtrigona (Oudemans, 1916) (625, 633, 663, 689, 709, 737, 740).

*S. vera (Moritz, 1964).

$\dagger$ Suctobelbella sp. (unidentified) (648) (one specimen).

Distribution: not applicable.

Superfamily Tectocepheoidea Grandjean, 1954

Family Tectocepheidae Grandjean, 1954 
Genus Tectocepheus Berlese, 1895

*^ Tectocepheus velatus (Michael, 1880) (618, 620, 623, 627, 648, 651, 661, 665, 668, 671, 674, 680, 682, 691, 693, 696, 698, 703, 707, 709, 712, 713, 723, 731, 737, 742, 744, 748, 751).

†Tectocepheus velatus alatus Berlese, 1913 (617, 622, 624, 638, 641, 662, 672, 690, 694, 702, 706, 710, 711, 714, 727, 741, 745, 746, 747) (numerous specimens).

Distribution: Palaearctic (Weigmann, 2006).

Superfamily Ameronothroidea Willmann, 1931

Family Ameronothridae Willmann, 1931

Genus Ameronothrus Berlese, 1896

${ }^{\wedge}$ Ameronothrus lineatus (Thorell, 1871) (679).

${ }^{\wedge}$ A. maculatus (Michael, 1882) (617, 643, 644, 658, 659, 664, 681, 684, 697, 708, 717, 728, 730, 736, 739, 749).

*^A. marinus (Banks, 1896) (679) (as Hygroribates marinus in Luxton, 1987).

^A. schneideri (Oudemans, 1903) (679).

Superfamily Licneremaeoidea Grandjean, 1954

Family Licneremaeidae Grandjean, 1954

Genus Licneremaeus Paoli, 1908

${ }^{\wedge}$ Licneremaeus licnophorus (Michael. 1882) (618).

Family Scutoverticidae Grandjean, 1954

Genus Scutovertex Michael, 1879

*^Scutovertex sculptus Michael, 1879 (624, 635, 653, 665, 667, 673, 676, 680, 681, 685, 690, 695, $696,698,717,731,733,735,747)$.

Superfamily Phenopelopoidea Petrunkevich, 1955

Family Phenopelopidae Petrunkevich, 1955

Genus Eupelops Ewing, 1917

${ }^{\wedge}$ Eupelops acromios (Hermann, 1804) (709).

${ }^{\wedge}$ E. hirtus (Berlese, 1916) (647).

${ }^{\wedge}$ E. plicatus (C.L. Koch, 1839) (621, 624, 625, 709, 677, 682, 711, 731, 743).

${ }^{\wedge}$ E. tardus (C.L. Koch, 1835) $(644,667,732)$.

${ }^{\wedge}$ E. torulosus (C.L. Koch, 1839) (640).

Genus Peloptulus Berlese, 1908

${ }^{\wedge}$ Peloptulus phaeonotus (C.L. Koch, 1844) (624, $650,663,665,674)$.

Superfamily Achipterioidea Thor, 1929

Family Achipteriidae Thor, 1929

Genus Achipteria Berlese, 1885

*^Achipteria coleoptrata (Linnaeus, 1758) (617, 644, 663, 671, 682, 721, 732).

${ }^{\wedge} A$. nitens (Nicolet, 1855) (623).

Genus Parachipteria van der Hammen, 1952

^ Parachipteria punctata (Nicolet, 1855) (623, 630, 631, 638, 664, 680, 700, 704, 711, 719, 723, 726, 729, 735, 742, 744, 749).

* P. willmanni van der Hammen, 1952.

Genus Pseudachipteria Travé, 1960

${ }^{\wedge}$ Pseudachipteria magna (Sellnick, 1928) $(628,634$, 635, 643, 672, 680, 681, 683, 689, 700, 720, 731, $733,734,735,748,749)$
Superfamily Oribatelloidea Jacot, 1925

Family Oribatellidae Jacot, 1925

Genus Joelia Oudemans, 1906

$\wedge$ Joelia fiorii (de Coggi, 1898) (622, 640, 740).

Genus Ophidiotrichus Grandjean, 1953

^Ophidiotrichus tectus (Michael, 1884) (619, 623, 636, 649, 672, 677, 691, 700, 707, 724, 736, 741).

Genus Oribatella Banks, 1895

^Oribatella quadricornuta (Michael, 1880) (647, 654, 674, 680, 686, 687, 689, 691, 696, 711, 731, $736,743)$

Superfamily Oripodoidea Jacot, 1925

Family Oribatulidae Thor, 1929

Genus Oribatula Berlese, 1895

†Oribatula longilamellata Schweizer, 1956 (680) (one specimen).

Distribution: Central Western Europe (Subías ,2019); Switzerland, Poland, Germany (Weigmann, 2006).

${ }^{\wedge} O$. tibialis (Nicolet, 1855) $(617,621,624,625,640$, 650, 656, 660, 662, 663, 667, 671, 672, 674, 676, 682, 685, 709, 715, 724, 733, 741, 749).

Genus Phauloppia Berlese, 1908

*^Phauloppia lucorum (C.L. Koch, 1841) (620, 629, 640, 644, 654, 659, 673, 675, 681, 684, 685, 690, 698, 714, 721, 728, 730, 731, 733, 739, 747, 749, 751).

Note: Luxton (1987) listed Phauloppia longiporosa as resident in the Isle of Man, but it is known to be a synonym of $P$. lucorum (Weigmann, 2006).

Genus Zygoribatula Berlese, 1916

*^Zygoribatula exilis (Nicolet, 1855) (699, 721, 729).

*Z. knighti Luxton, 1987 (subsequently found at Talacre Dunes, Wales: Monson, 2000).

$\dagger$ Zygoribatula propinqua (Oudemans, 1900) (696) (one specimen).

Distribution: Palaearctic (Weigmann, 2006).

Family Scheloribatidae Grandjean, 1933

Genus Liebstadia Oudemans, 1906

${ }^{\wedge}$ Liebstadia longior (Berlese, 1908) (695).

Note: Previously listed in Luxton (1996) as Protoribates badensis; see also Miko \& Weigmann (1996).

*^L. similis (Michael, 1888) $(619,624,627,636$, $638,648,650,651,655,657,661,663,666,667$, 669, 679, 680, 682, 685, 686, 689, 693, 697, 703, 708, 709, 710, 711, 719, 722, 727, 731, 732, 736, 747).

Genus Scheloribates Berlese, 1908

Subgenus Scheloribates (Hemileius) Berlese, 1916

${ }^{\wedge}$ Scheloribates (Hemileius) initialis (Berlese, 1908) $(617,621,622,627,638,662,663,674,680,682$, $693,732)$.

Subgenus Scheloribates (Scheloribates) Berlese, 1908

${ }^{\wedge}$ Scheloribates (Scheloribates) ascendens Weigmann \& Wunderle, 1990 (633, 643, 644, 659, 673, 679, 684, 717, 728).

*^S. (S.) laevigatus (C.L. Koch, 1836) (624) (as Scheloribates laevigatus in Luxton, 1987). 
${ }^{\wedge} S$. (S.) latipes (C.L. Koch, 1844) $(616,672,680$, 731).

$\uparrow$ Scheloribates (Scheloribates) minifimbriatus Mínguez, Subías \& Ruis, 1986 (704) (one specimen).

Distribution: Mediterranean and North Carolina, U.S.A. (Subías, 2019); Portugal (Weigmann, 2010).

Superfamily Ceratozetoidea Jacot, 1925

Family Ceratozetidae Jacot, 1925

Genus Ceratozetes Berlese, 1908

$\dagger$ Ceratozetes conjunctus Mihelčič, 1956 (704) (one specimen).

Distribution: Southern Palaearctic (Mediterranean area and Iran) (Subías, 2019).

${ }^{\wedge}$ C. gracilis (Michael, 1884) (617, 632, 663, 678, 682, 689, 694, 703).

*^C. peritus Grandjean, $1951(617,621,622,626$, 640, 664, 691, 693, 697, 703, 711, 732, 740, 748).

$\uparrow$ Ceratozetes sp. (unidentified) (672) (four specimens).

Distribution: not applicable.

Genus Edwardzetes Berlese, 1914

*^Edwardzetes edwardsi (Nicolet, 1855) (619, 624, 638, 641, 648, 664, 677, 693, 694, 704, 726, 742, 746).

Genus Fuscozetes Sellnick, 1928

*^Fuscozetes fuscipes (C.L. Koch, 1844) (655).

Genus Melanozetes Hull, 1916

^Melanozetes mollicomus (C.L. Koch, 1839) (624, 630, 638, 664, 683, 703, 704, 709, 715, 726, 735, 742, 744, 749).

${ }^{\wedge} M$. stagnatilis (Hull, 1914) $(664,742)$.

Genus Sphaerozetes Berlese, 1885

${ }^{\wedge}$ Sphaerozetes piriformis (Nicolet, 1855) (628, 634, 635, 700, 704, 711, 734, 735, 742, 749).

*S. orbicularis (C.L. Koch, 1835).

Genus Trichoribates Berlese, 1910

${ }^{\wedge}$ Trichoribates berlesei Jacot, 1929 (618, 625, 643, 644, 646, 686, 689, 698).

(= T. trimaculatus sensu Sellnick 1928 (Weigmann, 2006)).

${ }^{* \wedge} T$. incisellus (Kramer, 1897) $(667,669,685,686$, $727,738,743$ ) (as Latilamellobates incisellus in Luxton, 1987).

${ }^{\wedge} T$. novus (Sellnick, 1928) $(647,685,686,743)$.

Family Chamobatidae Grandjean, 1954

Genus Chamobates Hull, 1916

*^Chamobates borealis (Trägårdh, 1902) $(624,648$, 706, 710, 711, 713, 737).

*^C. cuspidatus (Michael, 1884) (630, 720, 634, 641, 646, 672, 678, 685, 693, 701, 720, 734).

${ }^{\wedge}$ C. interpositus Pschorn-Walcher, 1953 (706, 710, 739).

${ }^{\wedge}$ C. pusillus (Berlese, 1895) (618, 619, 622, 623, $625,627,630,631,632,633,636,637,638,639$, $640,645,647,649,651,652,654,656,661,662$, $664,670,672,674,677,680,682,683,685,690$, 694, 695, 699, 704, 705, 707, 710, 712, 713, 714, $716,719,720,721,722,724,727,729,731,737$, 739, 740, 741, 742, 743, 749).

Genus Ocesobates Aoki, 1965
^Ocesobates tumidisetosus (Monson, 2002) (648, 734)

Note: This species was referred to as Chamobates tumidisetosus by Monson (2002); Subías (2019) placed it in Ocesobates.

Family Euzetidae Grandjean, 1954

Genus Euzetes Berlese, 1908

*^Euzetes globulus (Nicolet, 1855) (617, 621, 622, 626, 634, 638, 640, 655, 666, 677, 682, 688, 691, $695,697,706,711,715,734,748)$.

Note: This species is generally referred to as E. globulus. However, as Hull (1920) has pointed out, E. nitens (Johnston, 1853), being earlier, should take precedence. The even earlier nomenclature suggested by some authors - E. seminulum (O.F. Müller, 1776) - has been dismissed by van der Hammen (1952) as being insufficiently founded (Luxton, 1990c).

Family Humerobatidae, Grandjean, 1971

Genus Humerobates Sellnick, 1928

*Humerobates rostrolamellatus Grandjean, 1936.

Family Punctoribatidae Thor, 1937 (= Mycobatidae Grandjean, 1954)

Genus Minunthozetes Hull, 1916

${ }^{\wedge}$ Minunthozetes semirufus (C.L. Koch, 1841) (687, 716, 737, 739).

Genus Mycobates Hull, 1916

${ }^{\wedge}$ Mycobates parmeliae (Michael, 1884) (644, 673, 684).

Genus Punctoribates Berlese, 1908

^Punctoribates punctum (C.L. Koch, 1839) (638, 674, 739, 747).

Genus Zachvatkinibates Shaldybina, 1973

${ }^{\wedge}$ Zachvatkinibates quadrivertex (Halbert, 1920) (679) (as Punctoribates quadrivertex

in Luxton, 1996).

Superfamily Galumnoidea Jacot, 1925

Family Galumnidae Jacot, 1925

Genus Acrogalumna Grandjean, 1956

^Acrogalumna longipluma (Berlese, 1904) (617, 621, 622, 626, 640, 697, 701, 711, 715, 731, 732, $740,748)$.

Genus Pergalumna Grandjean, 1936

^Pergalumna altera (Oudemans, 1915) (622).

\section{DISCUSSION}

Representatives of 172 species, one subspecies, 85 genera and 40 families have now been recorded from the Isle of Man. Included in the list is a species of Suctobelbella Jacot, 1937 and another of Ceratozetes Berlese, 1908 that are as yet unidentified and possibly new to science. All other species and subspecies found new to Britain in this collection are also recorded for mainland Europe (Weigmann, 2006; Subías, 2019).

The original collections made in the Isle of Man (Luxton, 1987) suggested that the oribatid fauna in this location is rather unusual. For instance, several of the new British records from there (e.g. Zygoribatula knighti, subsequently collected in Freshwater West, Dyfed (Luxton, 1987; Monson, 2000)) resemble more 
closely the fauna of the Iberian peninsula (and further east) than those of the rest of Britain. The Isle of Man is known to have been glaciated at the time of the last Ice Age (Manx Geological Society, 2020); whether species could have survived the glaciation is debatable. Due to under-recording, Mediterranean and eastern species may be more widely distributed across Western Europe than previously realised; the oribatid fauna of the Mediterranean region itself has been much better studied. However, it is also possible that transport through anthropogenic mechanisms (e.g. shipping) or some form of natural spread associated with climate change may be responsible for the appearance of these species.

The Isle of Man appears to have a very high species richness in comparison with other islands and archipelagos of the British Isles: Shetland, 100 (Monson, 2014); Orkney, 36 (Luxton, 1989); Holy Island (Northumberland), 15 (Luxton, 1990a); Jersey (Channel Isles), 67 (Luxton, 1990b); and Scilly Isles, 44 (Luxton, 1990c). The five most speciose families were Oppiidae (20 species), Brachychthonidae (16), Suctobelbidae (14), Ceratozetidae (13) and Euphthiracaridae (eight). The five most species-rich samples were 689 (Cass-ny-Hawin Head, gorse litter: 30 species), 649 (Tholt-e-Will Glen, heather litter: 29), 627 (Laxey Glen Gardens, beech litter: 28), 648 (Tholt-e-Will Glen, moss: 25) and 617 (The Raggett, larch litter: 21). The list will probably be incomplete since large areas of the island were not searched by M.L. (see Fig.1). In addition, samples were of fairly small size, each amounting to just a few handfuls of material. It might be useful for a future collector on the Isle of Man to visit farms (the older the better), particularly farm buildings and dutch barns containing damp hay and/or rotting straw. In this habitat on the mainland F.D.M. has found interesting species new to Britain, viz. Baioppia (Lalmoppia) talacrensis (Monson, 2000), Epidamaeus cf. floccusus (Behan-Pelletier and Norton, 1985), Haplozetes vindobonensis (Willmann, 1935) and Oppia denticulata (R. \& G. Canestrini, 1882) (Monson, in prep.).

The success of this survey will, it is hoped, be a stimulus for future work on the Isle of Man and elsewhere, with a view to furthering our knowledge of oribatids within a European context.

\section{ACKNOWLEDGEMENTS}

We are grateful to Professor R. Norton (State University of New York) for kindly reviewing the manuscript and to two reviewers for their constructive comments. The original survey was supported by Small Ecological Project Grant No. 590, British Ecological Society, 1988.

\section{REFERENCES}

Behan-Pelletier, V.M. \& Lindo, Z. (2019). Checklist of oribatid mites (Acari: Oribatida) of Canada and Alaska. Zootaxa 4666(1), 1-180.

https://doi.org/10.11646/zootaxa.4666.1.1
Hammen, L. van der (1952). The Oribatei of the Netherlands. Zoologische Verhandelingen, Leiden, 40, 1-93.

Halliday, R.B. \& Norton, R.A. (2019). Case 3785 Proposal to remove the homonymy of Cepheidae Berlese, 1896 (Acariformes) with Cepheidae Agassiz, 1862 (Cnidaria), by emending the former to Cepheusidae. Bulletin of Zoological Nomenclature 30, 39-46. https://doi.org/10.21805/bzn.v76.a010

Hull, J.E. (1920). An acarological pioneer. Vasculum 6, 95-102.

Luxton, M. (1987). Oribatid mites (Acari: Cryptostigmata) from the Isle of Man. The Naturalist 112, 67-77.

Luxton, M. (1989). Oribatid mites (Acari: Cryptostigmata) from Orkney. The Naturalist 114, 85-91.

Luxton, M. (1990a). Oribatid mites from Holy Island. Transactions of the Natural History Society of Northumbria 55(2), 144-146.

Luxton, M. (1990b). Oribatid mites (Acari: Cryptostigmata) from Jersey. Annual Bulletin Société Jersiaire 25(2), 360-366.

Luxton, M. (1990c). Oribatid mites (Acari: Cryptostigmata) from the Isles of Scilly. The Naturalist 115, 7-11.

Luxton, M. (1996). Oribatids of the British Isles: a check-list and notes on biogeography (Acari: Oribatida). Journal of Natural History 30, 803-822. https://doi.org/10.1080/00222939600770441

Manx Geological Survey (2020). Glacial sediments. https://www.manxgeology.com/glacial/ Accessed 16th March 2020.

Miko, L. \& Weigmann, G. (1996). Notes on the genus Liebstadia Oudemans, 1906 (Acarina, Oribatida) in Central Europe. Acta Musei Nationalis Pragae, Series B, Historia Naturalis 52(1-4), 73-100.

Monson, F.D. (2000). New and interesting oribatid mites (Acari: Oribatida) from Talacre Dunes, NorthEast Wales. The Naturalist 125, 149-166.

Monson, F.D. (2002). The oribatid mites (Acari: Oribatida) of Stockton's Wood, Speke Hall, Merseyside, including the description of a new Chamobates Hull, 1916 species. Journal of the Lancashire \& Cheshire Entomological Society 124126, 59-65.

Monson, F.D. (2011). Species of oribatid mite (Acari: Oribatida) new to Britain. British Journal of Entomology and Natural History 24, 57-68.

Monson, F.D. (2014). The oribatid mites (Acari: Oribatida) of Shetland. Shetland Naturalist 3(2), 3348.

Schatz, H., Behan-Pelletier, V.M., O'Connor, B.M. \& Norton, R.A. (2011). Suborder Oribatida van der Hammen, 1968. In: Zhang, Z.Q. (Editor). Animal biodiversity: an outline of higher level classification and survey of taxonomic richness. Zootaxa 3148, 141-148. https://doi.org/10.11646/zootaxa.3148.1.26

Subías, L.S. (2019). Listado sistemático, sinonímico y biogeográfico de los ácaros oribátidos (Acariformes, Oribatida) del mundo (excepto fósiles). Graellsia 60 
(número extraordinario), 3-305. (Updated pdf of original 2004 version).

https://doi.org/10.3989/graellsia.2004.v60.iExtra.21 8

Walter, D.E. \& Proctor, H.C. (2013). Mites: Ecology, Evolution and Behaviour. Life at a Microscale. (2nd edition). Springer, Dordrecht.

https://doi.org/10.1007/978-94-007-7164-2

Watson, H.C. (1852). Cybele Brittanica; or British Plants and their Geographical Relations. Vol. III. Longman \& Co., London.

Weigmann, G. (2006). Hornmilben (Oribatida). Die Tierwelt Deutschlands und der angrenzenden Merresteile. Part 76. Goecke \& Evers, Keltern.

Weigmann, G. (2010). Oribatid mites (Acari: Oribatida) from the coastal region of Portugal. IV. The genera Coronoquadroppia, Scheloribates, Haplozetes and Pilobates. Soil Organisms 82(3), 383-406. 